\title{
Comparison and evaluation of several models for fitting the frequency response of dispersive systems
}

\author{
J. Ross Macdonald ${ }^{\text {a) }}$ \\ Department of Physics and Astronomy, University of North Carolina, Chapel Hill, \\ North Carolina 27599-3255
}

(Received 22 July 2002; accepted 26 November 2002)

\begin{abstract}
Using both simulated and experimental data, detailed comparisons are made between the different physical interpretations and responses of several important models commonly employed for fitting and analyzing conductive-system data sets, such as those for ionic glasses. Those considered are one following directly from stretched-exponential temporal response, designated the Kohlrausch K0; several ones indirectly associated with such stretched-exponential response: the original modulus formalism (OMF) model and corrected modulus formalism (CMF) ones; and the ZC model, one whose real-part conductivity expression has been termed "universal dynamic response." In addition, several models involving dielectric dispersion, rather than resistive dispersion, are found to be less appropriate for the present data than are the CMF ones. Of the four main conductive-system models the CMF approach fits data for a wide variety of materials much better than do the others. The OMF is shown to be both experimentally and theoretically defective and leads to poor and inconsistent fitting results. The simple $\mathrm{ZC}$ model involves nonphysical low-frequency-limiting real-part conductivity response and is usually less appropriate even than the K0. High- and low-frequency expressions and fit results for the various dielectric elements are presented, along with discussion of characteristic, peak, and mean relaxation times for the various models, failing to confirm some proposed relations between these quantities suggested earlier. (C) 2003 American Institute of Physics. [DOI: 10.1063/1.1539092]
\end{abstract}

\section{INTRODUCTION}

There are currently four main approaches for analyzing dispersive frequency response data of ionic materials. Of these, three involve stretched-exponential correlationfunction temporal response and lead to different types of Kohlrausch frequency-response models, all ultimately derived from stretched-exponential correlation-function temporal response. ${ }^{1,2}$ The K0 model, conceptually the simplest, is just the Fourier transform of stretched-exponential response. ${ }^{3-8}$ Next is the original modulus formalism (OMF) of Moynihan and associates,,${ }^{9,10}$ and the third is the corrected modulus formalism (CMF). ${ }^{3,7,8,11-14}$ The fourth model, the $\mathrm{ZC}$, involves complex power-law behavior with an exponent $n$, and it is often designated as "universal dynamic response" (UDR) when only the real part of its conductivity is considered (Ref. 6) and references therein, Ref. 15.

Although prior work indicates that the CMF is both theoretically and experimentally more appropriate than the OMF and ZC/UDR approaches, ${ }^{6-8,16}$ both of the latter models continue to be widely used for data fitting and analysis. The present work includes new comparisons between these various models in order to help the reader pick the most appropriate one for future use. The four models, as well as several dielectric-dispersion ones, are defined and discussed in Sec. II, and some of their fitting results to experimental data are illustrated in Sec. III. Finally, Sec. IV compares formulas and fit results for mean values, peak values, and various dielectric constants calculable using these models.

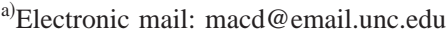

\section{SUMMARY OF VARIOUS RESPONSE MODELS}

\section{A. Kohlrausch response models and the OMF and CMF approaches}

Electrode effects and possible nearly-constant-loss (NCL) behavior ${ }^{16-19}$ are included here as appropriate, together with the basic fitting models discussed in the following. Let us use the subscript $k$, taken equal to $D, 0,1$, or $Z$, to distinguish some of the different types of dispersive frequency response models. Here $D$ specifies bulk dielectric response and $Z$ designates the $\mathrm{ZC}$ model. For $k=0$, define the stretched-exponential temporal response as

$$
\phi_{0}(t)=\exp \left[-\left(t / \tau_{o}\right)^{\beta_{0}}\right], \quad 0<\beta_{0} \leqslant 1,
$$

where $\tau_{o}$ is the characteristic relaxation time of the response. The 0 subscripts are changed in Eq. (1) to $D$ ones for dielectric situations. In most of the literature involving Kohlrausch (also designated by KWW or just $\mathrm{K}$ ) response models, no distinction has been made between the $K=D, 0$, and 1 values of the fractional exponent $\beta_{k}$, and it has usually been designated as just $\beta$, sometimes leading to ambiguity.

Next, define the normalized frequency response quantity ${ }^{2,3}$

$$
I_{k}(\omega) \equiv \frac{U_{k}(\omega)-U_{k}(\infty)}{U_{k}(0)-U_{k}(\infty)}=I_{k}^{\prime}-i I_{k}^{\prime \prime},
$$

so $I_{k}(0)=1$ and $I_{k}(\infty)=0$. For pure dielectric dispersion, ${ }^{2,20}$ $U_{D}(\omega) \equiv \epsilon(\omega)$, where $\epsilon(\omega)$ is the complex dielectric constant. For pure conductive-system dispersion set $k=0,1$, or $Z$ and $U_{k}(\omega) \equiv \rho(\omega)$, where $\rho(\omega)$ is the complex resistivity, equal to the inverse of the complex conductivity $\sigma(\omega)$ 
$=\sigma^{\prime}(\omega)+i \sigma^{\prime \prime}(\omega)$. Note that for conductive systems, the CMF and the OMF have usually implicitly or explicitly assumed that the quantity $\rho_{\infty} \equiv \rho(\infty)$ is zero, although the effects when it is small but nonzero have been discussed elsewhere. ${ }^{3,14}$ For simplicity and because $\rho_{\infty}$ cannot be determined by data fitting unless the data extend to very high frequencies, it will also be taken as zero herein.

For $k=D$ or 0 but not 1 , the normalized frequency response is given by ${ }^{2,10,13}$

$$
I_{k}(\omega)=\int_{0}^{\infty} \exp (-i \omega t)\left(-\frac{d \phi_{k}(t)}{d t}\right) d t .
$$

For the K0 Kohlrausch response model, the $\phi_{0}(t)$ used in Eq. (3) is that of Eq. (1) and is a conductive-system correlation function. The $k=D \phi_{D}(t)$ quantity is proportional to the dielectric transient response current, and the $I_{D}(\omega)$ frequency response is that of the KD model. ${ }^{2,20}$ Note that Eqs. (2) and (3) should be applied only for a single dispersive process. ${ }^{12}$ Thus, effects not directly associated with such dispersion are not then included in the response, and only ones arising entirely from mobile charge effects are involved herein for $k \neq D$.

The situation is a bit more complex for the conductivesystem K1 Kohlrausch response model, that appropriate when $k=1$ and used for both the OMF and CMF approaches. The OMF K1 analysis begins with the $\phi_{0}(t)$ quantity, defined as a conductive-system correlation function for electric field decay at constant dielectric displacement. ${ }^{10}$ This macroscopic approach, which also involves a quantity equivalent to $I_{0}(\omega)$, has come to be known as the original modulus formalism because it was first derived at the complex modulus level, where $M(\omega)=i \omega \epsilon_{V} \rho(\omega)=M^{\prime}(\omega)+i M^{\prime \prime}(\omega)$. Here $\epsilon_{V}$ is the permittivity of vacuum. A microscopic model with formally equivalent frequency response to the $\mathrm{K} 1^{13,14}$ was also published in $1973 ;^{21}$ see further discussion in Sec. III.

The corrected form of the K1 conductive-system modulus formalism, the CMF, when expressed at the modulus level, may be written as ${ }^{3-5,13,14}$

$$
M_{C 1}(\omega)=i \omega \epsilon_{V} \rho_{0} I_{1}(\omega)=\left[1-I_{0}(\omega)\right] / \epsilon_{C 1 \infty},
$$

where $I_{0}(\omega)$ follows from Eqs. (1) and (3) with $k=0, \rho_{0}$ $\equiv \rho(0)$, and $\epsilon_{C 1 \infty}$ is the high-frequency-limiting value of the conductive-system part of the dielectric constant, $\epsilon_{C 1}(\omega)$ $=1 / M_{C 1}(\omega)$. Here the subscript $C$ is used to denote the conductive-system response just as $D$ has been used to designate the bulk dielectric response, that present in the absence of mobile charges. The corresponding high-frequencylimiting bulk dielectric constant is $\epsilon_{D_{\infty}}$, and thus for conductive materials $\epsilon_{\infty} \equiv \epsilon_{C 1 \infty}+\epsilon_{D \infty}$, where $\epsilon_{\infty}$ is the highfrequency-limiting value of $\epsilon(\omega)$ for either the experimental data or for the total CMF theoretical model.

It is important to recognize that although in the modulus formalism the $I_{0}(\omega)$ appearing in Eq. (4) stems from stretched-exponential temporal response involving $\beta=\beta_{0}$, the $I_{1}(\omega)$ of this equation differs in form from $I_{0}(\omega)$, and thus its time-domain transform is not of stretchedexponential form, ${ }^{5,14}$ as is that of the $\mathrm{K} 0$ model. Therefore, when Eq. (4) is a part of a full K1 data fitting model with $\beta_{0}$ free to vary, fitting will lead to a different estimate of it than that which would have been obtained had the K0 model, where $M_{C 0}(\omega)=i \omega \epsilon_{V} \rho_{0} I_{0}(\omega)$, been used for fitting. The new $\beta$ estimate, arising from using the K1 model, is naturally termed $\beta_{1}$, and it is thus not appropriate to set $k=1$ in Eq. (3). Recent fitting results for CMF $\beta_{1}$ temperature, and ionic-concentration dependencies appear in Ref. 8 where $\beta_{1}$ is shown to be virtually independent of either variation.

Conductive-system analysis ${ }^{3,7,8,13,14}$ leads to the following important CMF general definitions of $\epsilon_{C 1 \infty}$, whose value may be estimated from data fitting as illustrated later:

$$
\begin{aligned}
\epsilon_{C 1 \infty} & =\sigma_{0} \tau_{o} /\left\langle x^{-1}\right\rangle_{1} \epsilon_{V}=\sigma_{0} \tau_{o}\langle x\rangle_{01} / \epsilon_{V} \\
& =\left[\gamma N(q d)^{2} / 6 k_{B} \epsilon_{V}\right] / T=A / T,
\end{aligned}
$$

where $\tau_{o}$ is, as usual, the characteristic relaxation time of the dispersion. Here $x \equiv \tau / \tau_{o}$, and so $\tau_{o}\langle x\rangle_{01}=\langle\tau\rangle_{01}$ and $\tau_{o}^{-1}\left\langle x^{-1}\right\rangle_{1}=\left\langle\tau^{-1}\right\rangle_{1}$. The quantity $\langle\tau\rangle$ is the mean of $\tau$ over the distribution of relaxation times of the dispersed response model. ${ }^{2,12,13}$ For the present Kohlrausch models, the 01 subscript indicates that $\langle x\rangle_{01}$ is the mean of $x$ over the K0 distribution involving $\beta_{1}$ rather than $\beta_{0}$, as implied by Eq. (4). The normalized means satisfy $\langle x\rangle_{01}=1 /\left\langle x^{-1}\right\rangle_{1}$ because of the close relation between the $\mathrm{K} 0$ and $\mathrm{K} 1$ distributions of relaxation times. ${ }^{3,12,22}$ Here $N$ is the maximum mobile charge number density; $\gamma$ is the fraction of charge carriers of charge $q$ that are mobile; and $d$ is the rms single-hop distance for the hopping entity. As usual we shall take the quantities in the square brackets of Eq. (5) temperature independent, so the parameter $A$ is then independent of temperature. ${ }^{8}$

Because there is always a contribution to the experimental high-frequency dielectric response from dipolar and vibronic bulk-material effects, it is insufficient to fit experimental data with a purely conductive-system response model, such as that of Eq. (4). For the usual frequency range employed for most measurements on ionic materials, roughly $10^{-2}-10^{6} \mathrm{~Hz}$, bulk dielectric dispersion is negligible, and, in the absence of ionic conduction, bulk response is adequately described by the frequency-independent dielectric constant $\epsilon_{D \infty}$. Although this quantity seems to increase somewhat with an increase in mobile charge concentration, ${ }^{8}$ for simplicity I shall follow common practice here and take it independent of frequency over the measured range as well as independent of ionic concentration.

The OMF equation corresponding to the CMF one of Eq. (4) is $3,10,23$

$$
M_{1}(\omega)=i \omega \epsilon_{V} \rho_{0} I_{1}(\omega)=\left[1-I_{0}(\omega)\right] / \epsilon_{\infty},
$$

differing only in the replacement of $\epsilon_{C 1 \infty}$ by $\epsilon_{\infty}$, where $\epsilon_{\infty}$ $\equiv \epsilon_{C 1_{\infty} \infty}+\epsilon_{D \infty}$. It thus accounts for the effects of $\epsilon_{D \infty}$ by implicitly combining conductive-system and dielectric responses in Eq. (6), improper because only the pure conductive-system Eq. (4)-model follows directly from Eqs. (1) to (3).

In contrast, the full CMF fitting approach must include the effect of $\epsilon_{D \infty}$ in a way more satisfactory than that of the $\mathrm{OMF}$, and thus it needs to involve a nondispersive dielectricresponse addition to the $\mathrm{K} 1$ model. The simplest way is to include a separate free fitting parameter, $\epsilon_{x}=\epsilon_{D \infty}$; the result- 
ing composite model is denoted CK1. It thus involves the conductive-system parameters $\sigma_{0}, \tau_{o}$, and $\beta_{1}$, as well as $\epsilon_{D \infty}$, and Eq. (5) allows an estimate of $\epsilon_{C 1 \infty}$ to be calculated when the value of $\langle x\rangle_{01}$ is known (see Sec. IV C).

A more general alternative model is to include in parallel with $\mathrm{K} 1$ a constant phase element (PCPE), defined at the complex dielectric level as

$$
\epsilon_{\mathrm{PC}}(\omega) \equiv A_{\mathrm{PC}}(i \omega)^{-\gamma_{\mathrm{PC}}},
$$

where $0 \leqslant \gamma_{\mathrm{PC}} \leqslant 1 .^{8,16,18,19}$ The resulting composite model, which can also represent nearly constant loss effects when present, is termed the PK1. Note that when $\gamma_{\mathrm{PC}}=0, \epsilon_{\mathrm{PC}}$ is an ordinary dielectric constant such as $\epsilon_{D \infty}$, and when $\gamma_{\mathrm{PC}}=1$ $\epsilon_{\mathrm{PC}}$ becomes a pure conductance. When $\epsilon_{\mathrm{PC}}$ is used to represent NCL behavior, $\gamma_{\mathrm{PC}} \ll 1$ and $A_{\mathrm{PC}} \approx \epsilon_{D \infty}$.

In contrast, the OMF fitting model involves only the $\sigma_{0}$, $\tau_{o}$, and $\beta_{1}$ parameters, allowing no separate estimation of $\epsilon_{D \infty}$ and $\epsilon_{C 1 \infty}$, but an estimate of $\epsilon_{\infty}$ may be calculated from the OMF analog of Eq. (5) $3,7,10,14,23,24$ using the abovegiven parameter estimates and

$$
\epsilon_{\infty}=\epsilon_{\mathrm{Ma}}\langle x\rangle_{01},
$$

where

$$
\epsilon_{\mathrm{Ma}} \equiv \sigma_{0} \tau_{o} / \epsilon_{V}
$$

is a Maxwell type of relation. In most applications of the OMF, instead of using Eq. (8) directly, Eqs. (8) and (9) are used to estimate $\sigma_{0}$ when an independent estimate of $\epsilon_{\infty}$ is available. Although the slightly greater simplicity of the OMF compared to the CMF encourages its use, OMF fitting invariably leads to inconsistencies in fitting experimental data and thus to much less accurate fitting than does the CMF. $^{6-8,14,16}$

In the past, there has been little direct fitting of Eq. (4) or (6) to data because no analytical result for the integral of Eq. (3) is available for arbitrary values of $\beta_{0}$ in Eq. (1). Therefore, $I_{0}(\omega)$ has had to be calculated numerically for each separate value of $\omega$ by a Fourier transform of Eq. (3), a task not amenable for data fitting with free model parameters. Luckily, an alternative exists and has been used in such data fitting by the author since 1996. Both the K0 and K1 frequency and temporal responses associated with such equations as Eqs. (4) and (6) may be accurately calculated or fitted using the free LEVM complex-nonlinear-least-squares computer program ${ }^{25}$ Further, unlike the Fourier transformation approach, LEVM allows possible inclusion in the total fitting model of not only K0 or K1 response but also of effects associated with $\epsilon_{D \infty}$, partial or full blocking at electrodes, and nearly constant loss. ${ }^{3,8,16-19}$

The $\beta_{0}$, OMF $\beta_{1}$, and CMF $\beta_{1}$ quantities associated with Eqs. (1), (4), and (6) are generally quite different and should not be designated by just $\beta$. The high-frequencylimiting $\log -\log \sigma^{\prime}(\omega)$ slopes of the three basic Kk models involving $\beta_{k}$ are $\left(1-\beta_{D}\right), \beta_{0}$, and $\left(1-\beta_{1}\right) .{ }^{4}$ Thus for a given conductive-system data set extending to sufficiently high frequencies we expect that $\beta_{0}$ and $\left(1-\beta_{1}\right)$ should both equal the ZC power-law exponent $n$. Some relevant fitting results are included in Sec. III.

\section{B. The $\mathrm{ZC}$ response model}

The ZC is probably the simplest useful response model. Its historical background is discussed in Ref. 6. At the complex conductivity level it may be expressed as

$$
\sigma_{Z}(\omega)=\sigma_{0}\left[1+\left(i \omega \tau_{Z}\right)^{n}\right],
$$

with $0<n \leqslant 1$. Although the ZC, particularly in a simplified expression for $\sigma_{Z}^{\prime}(\omega)$, the UDR response model, has been known and used for many years, ${ }^{6,15,26}$ both the power-law low-frequency-limiting behavior of $\left(\sigma_{Z}(\omega)-\sigma_{0}\right)$ and the high-frequency limiting response of $\sigma_{Z}(\omega)$ are nonphysical. Fitting of $\sigma^{\prime}(\omega)$ data to the $\sigma_{Z}^{\prime}(\omega)$ model thus invariably yields an inaccurate estimate of $\sigma_{0} \cdot{ }^{6}$ See also the fit results presented here in Sec. IV F.

The UDR form, defined at the real conductivity level, is

$$
\sigma_{Z}^{\prime}(\omega)=\sigma_{0}\left[1+\left(\omega \tau_{U}\right)^{n}\right]
$$

so it follows that $\tau_{U}=\tau_{Z}[\cos (n \pi / 2)]^{1 / n}$, generally smaller than $\tau_{Z}$. In the past, $\tau_{U}$ has been identified as the inverse of the hopping frequency of the charge carriers, ${ }^{27-30}$ but this interpretation was soon challenged ${ }^{26,31,32}$ and does not seem well justified either theoretically or experimentally. Further, recent work ${ }^{13,14}$ has shown that the Scher-Lax microscopic model $^{21}$ mean time for a hop can be identified as the CMF mean relaxation time $\langle\tau\rangle_{01}=\tau_{o}\langle x\rangle_{01}$ of the macroscopic CK1 model; see Eq. (5) and the discussion of the isomorphism of the microscopic and macroscopic models in Sec. III. Since there is no reason to believe that $\tau_{U} \simeq\langle\tau\rangle_{01}$, it should not be identified as the hopping time. Further, since the real and imaginary parts of Eq. (10) satisfy the KronigKramers relations, this equation should always be used in place of Eq. (11). ${ }^{3,6,33}$

When the $\mathrm{ZC}$ is used to analyze conductive-system data by means of complex-nonlinear-least-squares fitting, one must include the term $i \omega \epsilon_{V} \epsilon_{\infty}$ in the full fitting model at the complex conductivity level in order to account for the endemic presence of $\epsilon_{\infty}$. Note, however, that such a term contributes nothing to the $\sigma^{\prime}(\omega)$ part of the response. In the absence of electrode and NCL effects, it is clear from Eq. (10) that the high-frequency-limiting $\log -\log$ slope of $\sigma_{Z}^{\prime}(\omega)$ versus $\omega$ is just the exponent $n$. But limited-range data may not be sufficient to allow a good estimate of $n$ to be obtained unless the data are accurately described by Eq. (10) at high frequencies. If data are, as usual, well fitted by the CK1 or PK1 models, it is clear that at the complex admittance level the $\mathrm{C}$ of the CK1 approach should involve the term $i \omega \epsilon_{V} \epsilon_{D \infty}$, not the $i \omega \epsilon_{V} \epsilon_{\infty}$ of the ZC and CK0 models.

\section{COMPARISON OF SEVERAL FITTING MODELS}

\section{A. Preliminary comparisons}

In the first version of the present work, detailed comparison of fitting results of the same data sets using the OMF and CMF approaches was emphasized. A reviewer suggested that because "there is little doubt that there are electrical dipoles in ionic solids," such comparisons should include dielectricdispersion models as well as conductive-dispersion ones and cited the present Refs. 34-36 as illustrative of such comparison. These works dealt with data for melts, glasses, and liq- 
uid ionic solutions and assumed that dielectric dispersion associated with ion pairs was dominant and that no conductive-system dispersion was present.

The problem of deciding whether a given dispersive frequency response data set arises from mobile-charge effects (conductive-system dispersion) or from dielectric dispersion is an interesting one and was investigated in 1999 using accurate synthetic data. ${ }^{37}$ A K1 data set extending over a very wide frequency range was fitted with the $\mathrm{DK} 0 \equiv \mathrm{KD}$ model (and vice versa), where the " $\mathrm{D}$ " as usual denotes a dielectric dispersion situation. Because conductive-system response involves a nonzero $\rho(0)=1 / \sigma_{0}$ dc value, adequate dielectricsystem fitting of such data requires that a dc conductivity quantity be included in parallel with a pure dielectric response model, as in Refs. 34-36. Such a KD $\epsilon(\omega)$ model involves the four parameters $\epsilon_{\infty}, \Delta \epsilon \equiv \epsilon_{0}-\epsilon_{\infty}, \tau_{o D}$, and $\beta_{D}$, as in Eq. (2). Let us denote the inclusion of a parallel conductivity parameter by " $\mathrm{G}$ "; then an appropriate composite model may be designated by GDK0. Further, let " $\mathrm{S}$ " indicate the presence of a CPE term representing electrode effects, the SCPE, in series with a conductive or dielectric model. $8,16,18,19$ The number of free parameters in a composite fitting model will be included in parentheses after the name of the model, for example, GDK0S(7).

The isothermal comparisons of Ref. 37 showed that while one could generally well fit a conductive system involving resistive dispersion with one involving dielectric dispersion, and vice versa, such fits were not exact, allowing the different processes to be distinguished. When data are available for a range of temperatures, one would expect the activation energies of $\sigma_{0}$ and $\tau_{o}$ for a thermally activated conductive system to be the same or very nearly equal, ${ }^{8}$ while such behavior is unlikely for leaky dielectric situations. Since the authors of Ref. 38 found nearly equal activation energies using a dielectric-dispersion fitting model for lithium chloride solutions, it is likely that conductive-system analysis would have been more appropriate for their data. But this 1971 work was published before the OMF approach had been developed.

It is always a good idea to investigate the appropriateness of different fitting models when analyzing a new experimental data set. We shall here fit data for the single-crystal material $0.88 \mathrm{ZrO}_{2} \cdot 0.12 \mathrm{Y}_{2} \mathrm{O}_{3}$ at $T=503 \mathrm{~K} .{ }^{8}$ We consider conductive-system composite fits involving the $\mathrm{ZC}, \mathrm{K} 1$, and K0 models, and the dielectric-system ones GDK0, GDDC, and GDEXP ones as well. These dielectric-dispersion models were also used in Ref. 37. Here DC denotes the threeparameter Davidson-Cole model, and EXP stands for the asymmetrical exponential distribution-of-relaxations-times model. LEVM fitting employed proportional weighting of the complex data at the modulus level and led to values of the relative standard deviations of the fit residuals, $S_{F}$. For the ZC(3), CZC(4), and PZC(5) models, values found for $S_{F}$ were $0.243,0.0537$, and 0.0543 , respectively. All of these values are poor, but the $\mathrm{ZC}$ one led to completely inadequate parameter estimates.

Table I shows fitting results for 15 other models. No convergence could be obtained for fitting with the $\mathrm{K} 0(3)$ model since it involves no $\epsilon_{\infty}$ parameter. Note that both the
TABLE I. Comparison of values of $S_{F}$, the relative standard deviation of a fit, for 15 fits of experimental single-crystal $M(\omega)$ data of $0.88 \mathrm{ZrO}_{2}$ $\cdot 0.12 \mathrm{Y}_{2} \mathrm{O}_{3}$ at $T=503 \mathrm{~K}$. Numbers in parentheses denote the number of free fitting parameters present. Here an initial $\mathrm{C}$ in a model name indicates the presence of a capacitance or dielectric constant in parallel with the basic model; $\mathrm{P}$ indicates a parallel constant-phase-element; $\mathrm{S}$ indicates a series constant-phase-element; and GD indicates the presence of a conductance or conductivity in parallel with a dielectric dispersion model (see Sec. III A).

\begin{tabular}{lccccc}
\hline \hline K1 models & $S_{F}$ & K0 models & $S_{F}$ & GD models & \multicolumn{1}{c}{$S_{F}$} \\
\hline K1(3) & 0.071 & K0(3) & $\cdots$ & GDK1(5) & 0.094 \\
CK1(4) & 0.021 & CK0(4) & 0.035 & GDK0(5) & 0.019 \\
CK1S(6) & 0.0090 & CK0S(6) & 0.011 & GDDC(5) & 0.0133 \\
PK1S(7) & 0.0050 & PK0S(7) & 0.0057 & GDDCS(7) & 0.0101 \\
PK1(5) & 0.0049 & PK0(5) & 0.0083 & GDEXP(5) & 0.0116 \\
\hline \hline
\end{tabular}

OMF K1 model and the GDK1 one led to very poor fits. Further, although the $S_{F}$ values for the PK1S, PK0S, and GDDCS models are small, all these fits involved at least two free parameters with such large relative standard deviations that their values were statistically undetermined.

Although it is surprising that the bottom three K0-model fits led to $S_{F}$ values nearly as small as the corresponding K1 ones, it is clear that the PK1 model provided the best fit, as found earlier for these data, ${ }^{8}$ one very appreciably better than those of the GDDC and GDEXP. In addition, fits of the present data agree with the conclusion in Ref. 37 that a DEXP model yields a somewhat better dielectric-dispersion fit than does a DK0 or DDC one.

Another significant result found was that fitting with the GPK1 model led to essentially the same $S_{F}$ value as that for the PK1 but also to such a large uncertainty of the parallel conductivity parameter that it could not be statistically distinguished from zero. As mentioned in Ref. 37, when such a result appears it is a good indication that the data involve conductive dispersion rather than dielectric dispersion. The equality of the activation energies of $\sigma_{0}$ and $\tau_{o}$ for fits of data for the present material over a range of temperatures, ${ }^{8}$ as well as the present results, clearly indicate that these data sets involve resistive rather than dielectric dispersion. Nevertheless, some detailed comparisons between fittings by the two different approaches are included in the following.

\section{B. Detailed fitting results}

The results in Table I suggest that detailed GDEXPmodel fitting be compared to that obtained using the OMF $\mathrm{K} 1$ and the CMF PK1 models, but since the comparisons of Refs. 34-36 used the GDDC model, it will be employed here rather than the GDEXP one. Instead of using accurate complex-nonlinear-least-squares fitting of data to estimate OMF parameters, it has been customary for those employing the OMF approach to use the results of Table 2 in Ref. 10 to relate the width at half height of curves of $M^{\prime \prime}(\omega)$ data to $\beta\left(=\beta_{1}\right)$. As discussed in the following, this procedure yields inappropriate $\beta$ estimates. Alternatively, one may readily employ LEVM fitting to obtain accurate estimates of all OMF model parameters, as illustrated here.

Some detailed results of PK1, K1, and GDDC fittings of the data used for generating Table I are presented in Fig. 1. 


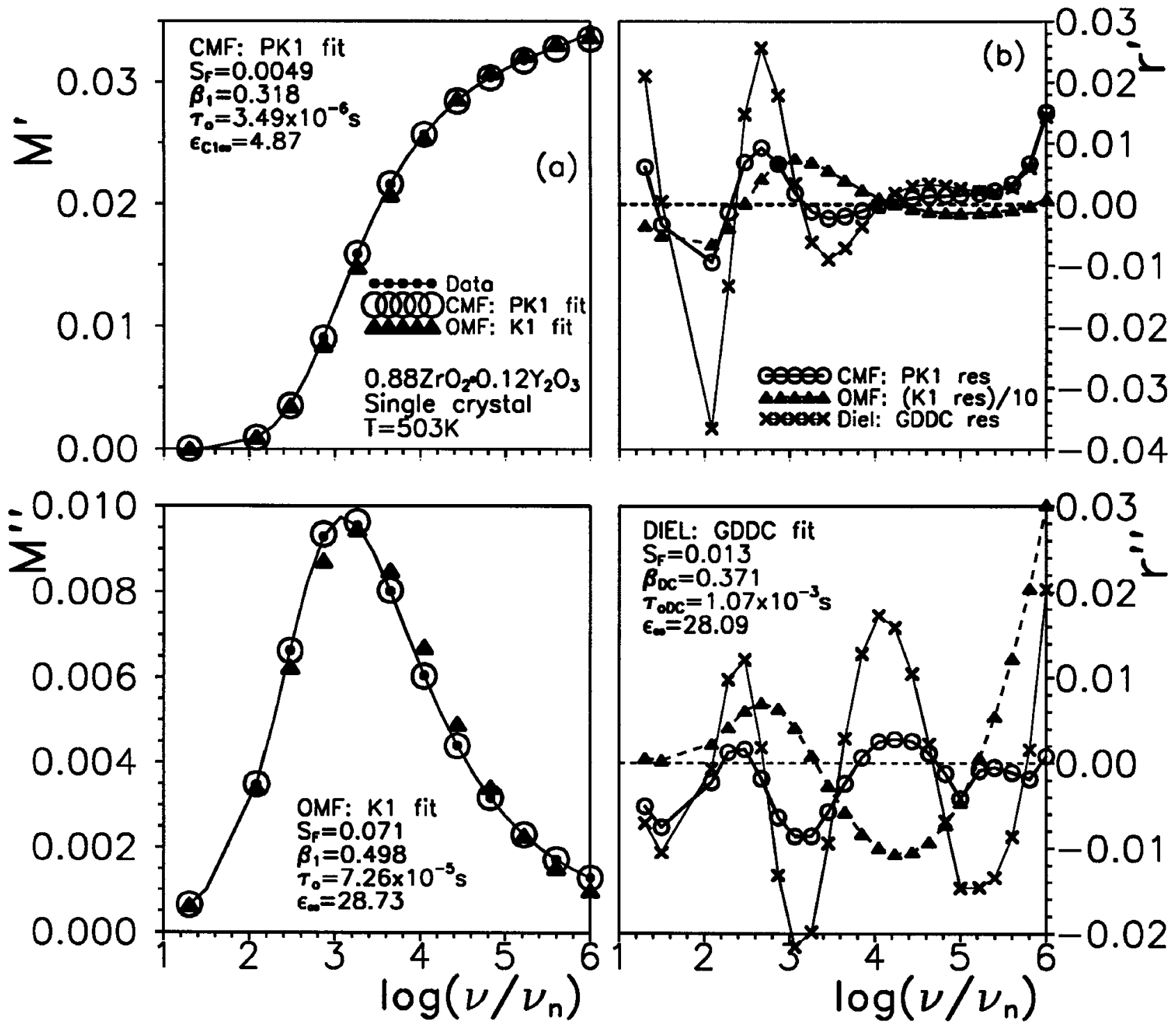

FIG. 1. (a) $M^{\prime}(\nu)$ and $M^{\prime \prime}(\nu)$ data and fit results for the PK1 and K1 models. (b) Real $\left(r^{\prime}\right)$ and imaginary $\left(r^{\prime \prime}\right)$ relative residuals, each defined here as (data-model prediction)/(model prediction), for PK1, K1, and GDDC model fits. The residual lines are included here solely to guide the eye. Here and hereafter, $\nu_{n}=1 \mathrm{~Hz}$. Note that the K1-fit residuals are shown here at one-tenth of their actual size.

Although the GDDC-fit points are omitted in Fig. 1(a) because they would not be well distinguishable, the differences between the three fit predictions are made clear by the residual plots of Fig. 1(b). These results, consistent with the corresponding $S_{F}$ values, show that the PK1 model is appreciably superior to the GDDC one, especially at low and midfrequencies, and both are far superior to the K1 model for fitting the present data.

Particularly important is the difference between the $\beta_{1}$ estimates shown. It arises from the absence of an $\epsilon_{D^{\infty}}$ parameter in the OMF K1 fit. Its $\epsilon_{C 1 \infty}$ estimate of 28.88 is identified, as usual in this approach, as $\epsilon_{\infty}$, and we see that it agrees quite well with the value from the GDDC fit. No $\epsilon_{\infty}$ estimate is shown for the PK1 fit because the presence of the PCPE term in this model, applying for all frequencies, prohibits the accurate determination of such a quantity. If, however, we approximate $\epsilon_{D \infty}$ by the $A_{\mathrm{PC}}=24.78$ PK1 fit estimate, not unreasonable since the associated $\gamma_{\mathrm{PC}}$ estimate is only 0.0039 , we obtain the value $\epsilon_{\infty} \simeq 29.65$, somewhat larger than the CK1S fit estimate of 28.29 where the $\beta_{1}$ estimate is 0.319. An improved PK1-like NCL approach is discussed in Ref. 8, and related work in progress that replaces the PCPE term by an effective-medium model does lead to a physically realizable expression for $\epsilon_{D_{\infty}}$.

Aside from the more accurate fit of the $\mathrm{CMF}$, true even when any CPE element is omitted, why should one prefer the $\mathrm{CMF}$ to the OMF? Further reasons are summarized in the following, but Fig. 2 makes the difference in fitting results graphic. Here we have plotted full CK1S and K1 $M^{\prime \prime}(\nu)$ fit results for comparison with the K1-only part of the CK1S and PK1 fits. The vertical dashed lines, plotted at $\nu_{o}$ $\equiv 1 / 2 \pi \tau_{o}$, clearly occur beyond the peaks of the curves, showing that the sometimes-used identification of $\nu_{o}$ with the peak frequency ${ }^{39}$ is inappropriate. Further, as shown here and hereafter, OMF estimates of $\tau_{o}$ are always much larger than are CMF-fit ones.

Although the K1 parameter estimates obtained from the CK1S and PK1 fits are in close agreement, the slightly 


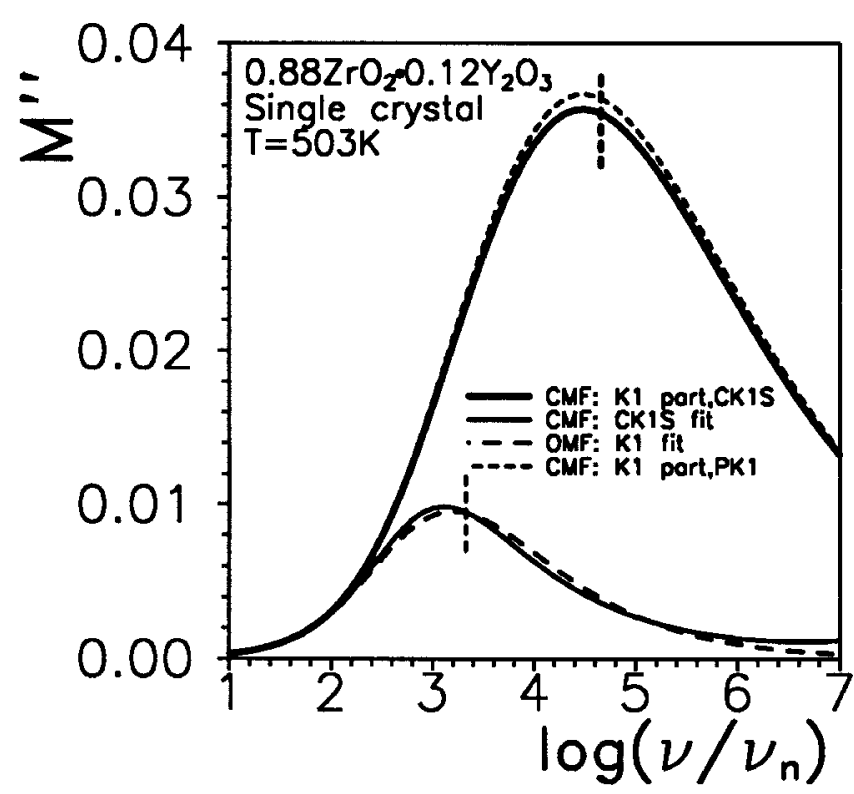

FIG. 2. Comparison of full OMF K1 and CMF CK1S fits of Fig. 1 data with the K1-response parts of the CMF CK1S and PK1 fits. The fit parameters were used to generate and extrapolate these results to higher frequencies. The vertical dashed lines show the positions of $\nu_{o} \equiv 1 / 2 \pi \tau_{o}$ for the OMF and CMF curves.

higher peak of the K1 part of the PK1 fit shown in Fig. 2 is associated with the lack of an exact $\epsilon_{D \infty}$ value for the PK1 fit, as discussed earlier. Nevertheless, the present results indicate that rather than just being arbitrary fitting models CK1S and PK1 fits allow one to closely estimate a K1-model description, one describing significant and meaningful physical response.

The difference between the CMF and OMF K1 curves is particularly large for the present data where $\epsilon_{D \infty} \gg \epsilon_{C 1 \infty}$, but the widths at half height of the top $\mathrm{K} 1$ curves of the figure nevertheless lead, on using Table 2 of Ref. 10, to $\beta_{1}$ estimates in close agreement with the direct-fit CMF ones of 0.319 and 0.318 for the CK1S and PK1, respectively. Reference 10, Table 2 actually applies only for the K1 model and should therefore never be used with experimental $M^{\prime \prime}(\omega)$ data because such data always include the effects of $\epsilon_{D \infty}$. Remember that the conductive-system K1 model and Eq. (4) are associated entirely with mobile-charge effects, and $\epsilon_{C 1 \infty}$ thus should not involve any bulk dipolar effects, such as those leading to $\epsilon_{D \infty}$.

Recent K1 CMF data analysis for different ionicconducting materials has indicated that $\beta_{1}$ is virtually independent of both temperature and mobile-ion concentration and is close to $1 / 3$ in value. ${ }^{7,8,16}$ This result is in agreement with an earlier study yielding $(1-n) \simeq 0.33$ estimates for a wide variety of materials, a study that also showed that OMF estimates of $\beta_{1}$ were quite different and of the order of $0.58 .^{40}$ Reference 40 also states that $(1-n) \simeq 0.33$ values are typically observed for mechanical losses in ionic glasses. Furthermore, nuclear spin relaxation results for a Li chloroborate glass analyzed by Eq. (1) with $k=0$ led to a value of $\beta_{0}$ of 0.35 , which was compared with an OMF electrical conductivity relaxation estimate of $0.50 .{ }^{41}$ These results begin to suggest that a $\mathrm{K} 1$ value of $\beta_{1}=1 / 3$ may possibly be a nearly universal result for various types of relaxation and materials.

The effect of limited frequency range on $\beta_{0}$ estimation was investigated starting with data for $\mathrm{Li}_{0.18} \mathrm{La}_{0.61} \cdot \mathrm{TiO}_{3}$ at $T=150 \mathrm{~K}$ kindly provided by Dr. C. León. LEVM fitting using the CMF approach led to parameter estimates that were then used to generate a virtually exact K1-model $M(\omega)$ data set with $\beta_{1}=1 / 3$ for the range $0.1 \leqslant \omega \leqslant 10^{8} r / s$. For this set, $\epsilon_{C 1 \infty}=2 / 3$. Since no value of $\epsilon_{D \infty}$ was included, the OMF and CMF approaches were formally equivalent. The data were then fitted with the CK0 model, one including a free $\epsilon_{x}$ parameter. It was needed to model the nonzero $\epsilon_{C 1 \infty}$ of the data since for the K0 $\epsilon_{C 0}(\infty) \equiv \epsilon_{C 0 \infty}=0$.

Fit results for both proportional and unity weighting should lead to $\beta_{0}=2 / 3$ if the relation $\beta_{0}=1-\beta_{1}$ were applicable. In fact, proportional-weighting fitting led to the large $S_{F}$ value of 0.073 and to the estimate $\beta_{0} \simeq 0.59$. With unity weighting, which emphasizes large data values, the fit at and near the peak was better and led to $\beta_{0} \simeq 0.61$. Better estimates of the limiting value were obtained by fitting just the $\sigma^{\prime}(\omega)$ data. Then, proportional and unity weighting led to estimates of 0.617 and 0.656 , respectively. When the data range was extended to $10^{9} \mathrm{r} / \mathrm{s}$, these estimates were improved to 0.628 and 0.667 , respectively.

As the above-mentioned results show, $\beta_{0}$ estimates from fits of synthetic K1 data are sensitive to the range of the data and to which immittance level is used in the fitting. For fits of experimental data with a CMF approach involving the $\mathrm{K} 1$ model, there is much less $\beta_{1}$ sensitivity for data with small random errors, and none of course when the data have vanishingly small random errors and are of $\mathrm{K} 1$ character.

In recent composite CMF K1 fits of limited-range data for several different materials, ${ }^{7,8,16}$ most $\beta_{1}$ estimates were again found to be close to $1 / 3$ for complex fits at any of the four immittance levels or for fits of any of the eight individual real or imaginary parts of the data. Such comparisons using the OMF K1 model for fitting were, however, inconsistent, and fits of $M(\omega)$ or $M^{\prime \prime}(\omega)$ yielded $\beta_{1}$ estimates much larger than the corresponding CMF ones. Inconsistency also appeared when OMF $\beta_{1}$ estimates for $\sigma^{\prime}(\omega)$ fits were compared with results for any of the other immittancelevel OMF fits. Since $\sigma^{\prime}(\omega)$ data values are independent of the presence or absence of $\epsilon_{D \infty}$ effects, OMF and CMF fits of data at this level should yield closely the same $\beta_{1}$ estimated values. Such results were indeed observed, verifying the appropriateness of the CMF and the inappropriateness of using the OMF for parameter estimation at the modulus level, as illustrated in Fig. 2 and in Sec. IV F.

A final inconsistency of the OMF approach appears when the formal results of the conductive-system microscopic continuous-time random-hopping model of Scher and $\mathrm{Lax}^{21}$ are compared to those of the macroscopic modulus formalism. ${ }^{13,14}$ The Scher-Lax model involves a [1 $\left.-I_{0}(\omega)\right]$ term in its response, just as in Eq. (4). The $\epsilon_{C 1}^{\prime \prime}(\omega)$ expressions following from the two models are of exactly the same form, but the $\epsilon_{C 1}^{\prime}(\omega)$ expressions differ by the absence of a nonzero $\epsilon_{C 1}^{\prime}(\infty)=\epsilon_{C 1 \infty}$ in the microscopic model. Note that with the present stretched-exponential expression for 
TABLE II. Fitting results using row- 1 synthetic $M(\omega)$ data derived from fitting single-crystal $0.88 \mathrm{ZrO}_{2} \cdot 0.12 \mathrm{Y}_{2} \mathrm{O}_{3}$ at $T=503 \mathrm{~K}$. In row 1 , $\epsilon_{D \infty}=23$, and it is zero for row 2. The dimension of $\sigma_{0}$ is S/cm and that of all $\tau$-related quantities is seconds. Rows 3 and 4: $M(\omega)$ fits; rows 5-7: $\sigma^{\prime}(\omega)$ fits. See Eqs. (23) $-(22)$ for $\langle x\rangle \equiv\langle\tau\rangle / \tau_{o}$ expressions.

\begin{tabular}{|c|c|c|c|c|c|c|c|c|c|}
\hline No. & Model & $100 S_{F}$ & $\beta_{k}$ & $10^{8} \sigma_{0}$ & $100 M_{p}^{\prime \prime}$ & $10^{4} \tau_{o}$ & $10^{4} \tau_{p}$ & $10^{4}\langle\tau\rangle_{01}$ & $10^{4}\langle\tau\rangle_{k}$ \\
\hline 1 & $\begin{array}{c}\text { CMF:CK1 } \\
\text { Data }\end{array}$ & $\cdots$ & $1 / 3$ & 1.845 & 1.035 & 0.0400 & 1.212 & 0.2400 & 2.400 \\
\hline 2 & K1 data & $\cdots$ & $1 / 3$ & 1.845 & 3.738 & 0.0400 & 0.0598 & 0.2400 & 2.400 \\
\hline 3 & OMF:K1 fit & 2.87 & 0.5460 & 1.850 & 1.017 & 0.8101 & 1.069 & 1.395 & 3.443 \\
\hline 4 & CK0 fit & 1.28 & 0.5233 & 1.841 & 1.020 & 1.096 & 1.186 & $\ldots$ & 2.023 \\
\hline 5 & CK0 $\sigma^{\prime}$ fit & 0.98 & 0.5402 & 1.865 & $\ldots$ & 1.017 & $\ldots$ & $\ldots$ & 1.781 \\
\hline 6 & $\begin{array}{c}\mathrm{ZC} \sigma^{\prime} \text { fit } \\
\sigma_{0} \text { free }\end{array}$ & 1.75 & 0.3898 & 1.716 & $\cdots$ & 1.093 & $\cdots$ & $\cdots$ & 3.905 \\
\hline 7 & $\begin{array}{c}\mathrm{ZC} \sigma^{\prime} \text { fit } \\
\sigma_{0} \text { fixed }\end{array}$ & 3.68 & 0.3492 & 1.845 & $\cdots$ & 0.8967 & $\cdots$ & $\cdots$ & 4.544 \\
\hline
\end{tabular}

$\phi_{0}(t)$ no overt Coulomb interactions appear in the microscopic (or macroscopic) K1 response model.

Unfortunately, the Kronig-Kramers relations do not lead to clarification of the above-mentioned difference in the two models, a difference ascribed by Scher (private communication) as arising from the inapplicability of the microscopic approach at very high frequencies. It has been shown, however, that a distribution of relaxation times estimated using LEVM from specific $\epsilon_{C 1}^{\prime \prime}(\omega)$ K1 response alone may be employed to estimate the corresponding $\epsilon_{C 1}^{\prime}(\omega)$ response. ${ }^{12,14}$ The resulting $\epsilon_{C 1}^{\prime}(\omega)$ response agreed well with the macroscopic-model response and included a proper nonzero value of $\epsilon_{C 1 \infty}$, showing that when the real and imaginary parts of the microscopic response are made consistent the two models are fully isomorphic. Since these calculations and responses involve only mobile-charge effects, the OMF is intrinsically nonisomorphic because its limiting dielectric constant involves dipolar as well as monopolar effects and the model is thus not isomorphic to the Scher-Lax microscopic response theory as claimed earlier. ${ }^{42,43}$

The above-mentioned CMF isomorphism provides an instructive microscopic interpretation of the K1 conductivesystem model. The Scher-Lax approach is that of stochastic hopping transport of charge involving a continuous-time random walk on a lattice. The excellent fits of experimental data using such macroscopic CMF models as the CK1, the CK1S, and the PK1 suggest that not only is one dealing with a conductive system but the physical processes associated with charge motion in the material investigated are well described by those of the microscopic Scher-Lax model.

The major problem with the OMF approach, the unwarranted replacement of $\epsilon_{C 1 \infty}$ by $\epsilon_{D \infty}$ or $\epsilon_{\infty}$, was first pointed out in $1994 .{ }^{11}$ As already mentioned, in 1995 Sidebottom, Green, and Brow ${ }^{40}$ showed that UDR power-law data analysis for a wide variety of materials yielded $(1-n)$ values of about 0.33 , quite different from their larger OMF $\beta_{1}$ estimates of about 0.58 , but no explanation of the difference was presented. Since then, the OMF and CMF data fitting approaches have been compared in detail. ${ }^{3-8,12,14}$ When electrode and/or nearly-constant-loss effects are properly accounted for, the OMF $\beta_{1}$ is found to increase with temperature, quite different from CMF-fit constant estimates of about $1 / 3 .^{8}$

\section{COMPARISONS OF MEAN-VALUE AND DIELECTRIC-CONSTANT EXPRESSIONS AND FIT RESULTS}

\section{A. Background}

Because all dispersed-response models lead to relations such as Eqs. (5) and (8) that involve averages over their distributions of relaxation times, it is important to compare means for various models as well as the resulting expressions that relate limiting dielectric constants and dc conductivity. We shall start with general relations for limiting dielectric constants and then show explicit forms and fitting results for the present models of interest. Denote the normalized relaxation-time distribution as $G(x)$. Then, generally ${ }^{2-6,12,13}$

$$
\begin{aligned}
\left\langle\tau^{m}\right\rangle_{k} & =\tau_{o}^{m}\left\langle x^{m}\right\rangle_{k}=\tau_{o}^{m} \int_{0}^{\infty} x^{m} G_{k}(x) d x \\
& =\left[\tau_{o}^{m} / \Gamma(m)\right] \int_{0}^{\infty} u^{m-1} \phi_{k}(u) d u,
\end{aligned}
$$

where $u \equiv t / \tau_{o}$ and $\Gamma(m)$ is the Euler gamma function. For $\mathrm{K} k$ conductive-system models, $G_{1}(x)=\left(x /\langle x\rangle_{01}\right) G_{0}(x) .^{3}$ Unfortunately, for the $k=Z$ ZC model with its nonphysical limiting responses, $\langle\tau\rangle_{Z}$, for example, does not exist unless $G_{Z}(x)$ is cutoff at both extremes. All the following results involve no cutoffs, ${ }^{5}$ appropriate for the data set used for the present comparisons. The quantity $\boldsymbol{\epsilon}_{x}$ is a free dielectric parameter used in fitting with some of the following models.

\section{B. CKO model}

$$
\begin{aligned}
& \epsilon_{C 00} / \epsilon_{\mathrm{Ma}}=\langle x\rangle_{0}=\beta_{0}^{-1} \Gamma\left(\beta_{0}^{-1}\right), \\
& \epsilon_{C 0 \infty} / \epsilon_{\mathrm{Ma}}=1 /\left\langle x^{-1}\right\rangle_{0}=0, \\
& \epsilon_{\infty}=\epsilon_{x}, \quad \epsilon_{0}=\epsilon_{C 00}+\epsilon_{\infty} .
\end{aligned}
$$

\section{CK1 CMF model}

$$
\begin{aligned}
& \epsilon_{C 10} / \epsilon_{\mathrm{Ma}}=\langle x\rangle_{1}=\left[\left\langle x^{2}\right\rangle_{01} /\langle x\rangle_{01}\right]=\Gamma\left(2 \beta_{1}^{-1}\right) / \Gamma\left(\beta_{1}^{-1}\right), \\
& \epsilon_{C 1 \infty} / \epsilon_{\mathrm{Ma}}=1 /\left\langle x^{-1}\right\rangle_{1}=\langle x\rangle_{01}=\beta_{1}^{-1} \Gamma\left(\beta_{1}^{-1}\right) \\
& \epsilon_{D \infty}=\epsilon_{x}, \quad \epsilon_{\infty}=\epsilon_{C 1 \infty}+\epsilon_{D \infty}, \quad \epsilon_{0}=\epsilon_{C 10}+\epsilon_{D \infty}
\end{aligned}
$$


TABLE III. Results for various dielectric quantities calculated from exact data (rows 1 and 2) and fits to the row-1 data (rows 3-7). Here $\epsilon_{\mathrm{Ma}}$ is the Maxwell-type quantity of Eq. (9). See Eqs. (13)-(23) for calculation formulas employed. The $\epsilon_{C Z 0}$ results are discussed in the text.

\begin{tabular}{lccccccc}
\hline \hline No. & Model & $\epsilon_{x}$ & $\epsilon_{\text {Ma }}$ & $\epsilon_{C k \infty}$ & $\epsilon_{\infty}$ & $\epsilon_{C k 0}$ & $\epsilon_{k 0}$ \\
\hline 1 & CMF: CK1 & 23 & $5 / 6$ & 5 & 28 & 50 & 73 \\
2 & K1 & $\ldots$ & $5 / 6$ & 5 & 5 & 50 & 55 \\
3 & OMF:K1 fit & $\ldots$ & 16.93 & 29.15 & 29.15 & 71.94 & 71.94 \\
4 & CK0 fit & 28.76 & 22.79 & 0 & 28.76 & 42.06 & 70.82 \\
5 & CK0 $\sigma^{\prime}$ fit & $\ldots$ & 21.42 & 0 & $\ldots$ & 37.51 & $\ldots$ \\
& $\sigma_{0}$ free & $\ldots$ & 21.21 & $\ldots$ & $\ldots$ & 74.44 & $\ldots$ \\
6 & ZC $\sigma^{\prime}$ fit & $\ldots$ & & $\ldots$ & $\ldots$ & 91.37 & $\ldots$ \\
7 & $\sigma_{0}$ free & $\ldots$ & 18.46 & $\ldots$ & $\ldots$ & & \\
\hline
\end{tabular}

\section{K1 OMF model}

$$
\begin{aligned}
& \epsilon_{10} / \epsilon_{\mathrm{Ma}}=\langle x\rangle_{1}=\left[\left\langle x^{2}\right\rangle_{01} /\langle x\rangle_{01}\right]=\Gamma\left(2 \beta_{1}^{-1}\right) / \Gamma\left(\beta_{1}^{-1}\right), \\
& \epsilon_{1 \infty} / \epsilon_{\mathrm{Ma}}=1 /\left\langle x^{-1}\right\rangle_{1}=\langle x\rangle_{01}=\beta_{1}^{-1} \Gamma\left(\beta_{1}^{-1}\right), \\
& \epsilon_{\infty}=\epsilon_{1 \infty}, \quad \epsilon_{0}=\epsilon_{10}+\epsilon_{\infty} .
\end{aligned}
$$

Although the right-hand sides of Eqs. (16) and (17) are the same in form as those of Eqs. (19) and (20), large differences between the $\tau_{o}$ and $\beta_{1}$ estimates obtained from $\mathrm{CMF}$ and OMF fits of the same data ensure that their $\epsilon_{C 10}$ and $\epsilon_{C 1 \infty}$ estimates will also differ substantially, as illustrated in Sec. IV F. Note that the conductive-system " $C$ " subscript is omitted here from the above-mentioned OMF dielectric-constant designations because the OMF approach is a combination of both conductive and dielectric responses, as already discussed.

\section{E. ZC model with $\beta_{Z} \equiv 1-n \simeq \beta_{0}$}

$$
\begin{aligned}
& \epsilon_{C Z 0} / \epsilon_{\mathrm{Ma}} \doteq\langle x\rangle_{0}=\int_{0}^{\infty} \phi_{0}(u) d u=\int_{0}^{\infty} \exp \left(-u^{\beta_{Z}}\right) d u \\
& =\beta_{Z}^{-1} \Gamma\left(\beta_{Z}^{-1}\right), \\
& \epsilon_{0}=\epsilon_{C Z 0}+\epsilon_{\infty} .
\end{aligned}
$$

Equation (22) for $\epsilon_{C Z 0}$, involving a Kohlrausch response approximation, is discussed in the following.

\section{F. Synthetic data fitting results}

In the following two tables, results are presented for fits of the various models to an exact CK1 data set. This set, which involved 81 points logarithmically distributed over the range $100 \leqslant \omega \leqslant 10^{6} \mathrm{r} / \mathrm{s}$, was derived by starting with the CK1S fit parameters, adjusting the values of those involving the CK1 part of the response slightly, and then using them in LEVM to generate the data. Thus, the resulting data set well represents the response of single-crystal $0.88 \mathrm{ZrO}_{2}$ $\cdot 0.12 \mathrm{Y}_{2} \mathrm{O}_{3}$ at $T=503 \mathrm{~K}$ with electrode or nearly-constantloss effects removed. Because LEVM yields the most accurate response for $\beta_{1}=1 / 3$ and because many fit results well approximate this value, it was used, along with $\rho_{0}=5.421$ $\times 10^{7} \Omega \mathrm{cm}$.
Table II presents values of various quantities for the exact data in rows 1 and 2 and for fit results in rows 3-7. Here $M_{p}^{\prime \prime}\left(\omega_{p}\right)$ is the peak value of the $M^{\prime \prime}(\omega)$ data or fit and $\tau_{p}$ $\equiv 1 / \omega_{p}$ is the corresponding tau value, where $\omega_{p}$ is the mode of the response curve. The $\tau_{p}$ column has been included because it was stated some years ago that for conductive systems $\tau_{p}$ and the $\langle\tau\rangle$ defined by $\tau_{p} \beta^{-1} \Gamma\left(\beta^{-1}\right)$ $=\epsilon_{V} \epsilon_{\infty} / \sigma_{0}$ were in close agreement. ${ }^{39}$ But because the results in Table II show that $\tau_{p} \neq \tau_{o}$, this definition of $\langle\tau\rangle$ is incorrect and unequal to that following from Eqs. (8) and (19) for the OMF, $\langle\tau\rangle_{01}=\tau_{o}\langle x\rangle_{01}$.

Table II also shows that there are no equalities between the estimates of $\tau_{o}, \tau_{p}$, and $\langle\tau\rangle_{01}$ obtained from the same fit results. León, Lucia, and Santamaria found excellent agreement, however, between their $\mathrm{ZC} \tau_{Z}=\tau_{o}$ and $\langle\tau\rangle_{01}$ estimates from fits of data for the same material as that considered here but one with a slightly smaller $\mathrm{Y}_{2} \mathrm{O}_{3}$ concentration. ${ }^{33}$ Such close agreement suggested to them that the two quantities might be the same. As in the present work, their $\langle\tau\rangle_{01}$ estimates involved the OMF K1 model, but their $\beta\left(=\beta_{1}\right)$ and other parameter estimates were derived by a series of approximations rather than directly as here. Here, the corresponding estimates for comparison are $\tau_{Z} \cong 1.093 \times 10^{-4} \mathrm{~s}$ and $\langle\tau\rangle_{01} \cong 1.395 \times 10^{-4} \mathrm{~s}$. Interestingly, much closer agreement is apparent here between the $\tau_{Z}$ estimate and the OMF $\mathrm{K} 1 \tau_{p} \cong 1.069 \times 10^{-4} \mathrm{~s}$ one. Accurate fitting of data from different materials and at different temperatures is needed in order to assess the generality of these results although the more significant comparisons are those involving $\mathrm{CMF}$ rather than OMF fitting results.

Table II shows $\sigma^{\prime}(\omega)$ fit results in rows 6 and 7 for the ZC model. These results therefore are also ones that would be obtained from a UDR-model fit using Eq. (11) except for the difference between $\tau_{Z}$ and $\tau_{U}$ estimates already mentioned. The results in rows 6 and 7 differ because $\sigma_{0}$ was, as usual, a free fitting parameter for the row- 6 fit and was held fixed at the exact row- 1 value for the row- 7 fit. Here $\beta_{Z}$ is defined as $(1-n)$, so its row-6 value corresponds to $n$ $\simeq 0.61$, a common value for UDR fits for materials and temperatures where electrode and NCL effects are negligible ${ }^{6,15,26,41}$ or properly accounted for in complexnonlinear-least-squares fitting. The row- 6 estimate of $\sigma_{0}$ is the worst of those in Table II, and when it is held fixed at the 
proper value, as in row 7, $S_{F}$ is far larger. The row-6 $\sigma_{0}$ estimate is poorest because ZC low-frequency-limiting behavior is physically improper. For this reason, in the absence of physically realizable cutoff, $\epsilon_{Z}(\omega)$ increases indefinitely as $\omega \rightarrow 0$ and $\langle x\rangle_{Z}$ is infinite.

Now it is clear that the ZC model is more comparable to the $\mathrm{K} 0$ rather than to the $\mathrm{K} 1$ one. For illustrative purposes only, I make the approximation of using $\mathrm{ZC}$ fit parameter estimates in the $\mathrm{K} 0$ expression of Eq. (22) in order to calculate the $\langle\tau\rangle_{Z} \doteq\langle\tau\rangle_{0}$ results of rows 6 and 7. It is clear that they are appreciably larger than the other values in Table II, perhaps in part because the $\beta_{Z}=(1-n)=\beta_{0}$ relation is too approximate.

When one carries out a $\sigma^{\prime}(\omega)$ OMF K1 fit of the present data, the fit is perfect and leads, as expected, to the results shown in row 2 . Note that the row-2 data and parameters are very close to those of the CMF top K1 curves of Fig. 2. Comparison of the row- 2 and row- 3 results thus illustrates the inconsistency of the OMF approach at its starkest, as do the Fig. 2 curves. Such inconsistency also leads to the large row-3 $S_{F}$ value. Comparison of the CK0 results of rows 4 and 5 indicates that although the comparable parameter estimates and $\langle\tau\rangle_{0}$ values are not exactly the same, they are reasonably close, and the $S_{F}$ values are much smaller than that of the row-3 OMF fit. The K0 model is thus appreciably more applicable here than is the OMF one, and differences between the parameter estimates of rows 4 and 5 are associated with the systematic errors arising from fitting CK1 data with a CK0 model, not from a basic inconsistency like that of the OMF model.

The results shown in Table III used those of Table II and Eqs. (13)-(23). Comparison of the values shown in rows 1 and 3 makes it clear that although the OMF K1 approach leads to estimates of $\epsilon_{\infty}$ and $\epsilon_{0}$ in reasonable agreement with those of the input CMF CK1 model, these quantities are formed in different ways. In particular, note that the OMF estimate of $\epsilon_{\infty}$ arises entirely from the $\epsilon_{C 1 \infty}$ estimate of the fit but includes the $\epsilon_{D \infty}$ value in this estimate, thus improperly combining both conductive and dielectric system quantities in this conductive-system parameter. Similar differences appear for the $\epsilon_{C 10}$ estimates.

It is evident that although the K0 fit cannot lead to separation of $\epsilon_{C 1 \infty}$ and $\epsilon_{D \infty}$ values because $\epsilon_{C 0 \infty}$ is zero or negligible, $M(\omega) \mathrm{K} 0$ fits nevertheless yield a better estimate of $\epsilon_{C 10}$ than do such fits using the OMF K1 model. Since $\sigma^{\prime}(\omega)$ fits lead to no $\epsilon_{\infty}$ estimates, no $\epsilon_{k 0}$ values are shown for rows $5-7$, but it is clear that the present analysis of ZC-fit data using a $\mathrm{K} 0$ expression yields very poor $\epsilon_{C 10}$ estimates and would yield far too large estimates of $\epsilon_{0}$. Finally, it is worth emphasizing that the conventional use of Eq. (20) of the OMF K1 model for estimation of $\sigma_{0}$ when a value of $\epsilon_{\infty}$ is known (or sometimes vice versa), is inappropriate for two reasons. First, data rarely extend to such high frequencies that an accurate, limiting frequency-independent estimate of $\epsilon_{\infty}$ is available. Second, the inconsistent, theoretically improper OMF approach should be superceded by the CMF approach, one that, through the use of LEVM or an equivalent fitting procedure, yields simultaneous accurate estimates of all pertinent parameters: $\beta_{1}, \tau_{o}, \sigma_{0}$, and $\epsilon_{D \infty}$, and so it then allows the calculation of valid estimates of $\epsilon_{C 1 \infty}$ and $\epsilon_{C 10}$.

Unfortunately, all publications dealing with the CMF have been largely ignored so far, and the OMF continues to be widely employed. For example, Ref. 41 provides a list of 20 OMF papers, many appearing after 1995, and many others continue to be published. Because science involves the search for truth, continuing users of the OMF should either accept the CMF or show where and why it is incorrect. Since neither has happened in the last seven years, it seems highly probable that the basic premise of the CMF is unlikely to be false and thus the CMF is a far more appropriate idealization of the actual conductive-system physical situation than is the OMF.

\section{ACKNOWLEDGMENTS}

It is a pleasure to thank Dr. Carlos León and Dr. Kia Ngai for stimulating discussions relevant to the area of the present work.

${ }^{1}$ R. Kohlrausch, Pogg. Ann. der Phys. und Chemie, (2) 91, 179 (1854); G. Williams and D. C. Watts, Trans. Faraday Soc. 66, 80 (1970).

${ }^{2}$ C. P. Lindsey and G. D. Patterson, J. Chem. Phys. 73, 3348 (1980).

${ }^{3}$ J. R. Macdonald, J. Non-Cryst. Solids 212, 95 (1997); 220, 107 (1997). In addition, the symbol $\sigma$ should be removed from the right end of Eq. (12) and " $d x$ " should be moved from the left side of the term in square brackets in Eq. (A5) to the right side.

${ }^{4}$ J. R. Macdonald, J. Appl. Phys. 82, 3962 (1997).

${ }^{5}$ J. R. Macdonald, J. Appl. Phys. 84, 812 (1998).

${ }^{6}$ J. R. Macdonald, Solid State Ionics 133, 79 (2000).

${ }^{7}$ J. R. Macdonald, J. Appl. Phys. 90, 153 (2001). In Eq. (10), $\sigma_{0} \Gamma$ should be replaced by $\sigma_{0} \tau_{o} \Gamma$

${ }^{8}$ J. R. Macdonald, J. Chem. Phys. 116, 3401 (2002).

${ }^{9}$ P. B. Macedo, C. T. Moynihan, and R. Bose, Phys. Chem. Glasses 13, 171 (1972).

${ }^{10}$ C. T. Moynihan, L. P. Boesch, and N. L. Laberge, Phys. Chem. Glasses 14, 122 (1973).

${ }^{11}$ B. A. Boukamp and J. R. Macdonald, Solid State Ionics 74, 85 (1994).

${ }^{12}$ J. R. Macdonald, J. Non-Cryst. Solids 197, 83 (1996); 204, 309 (1996). In addition, $G_{D}$ in Eq. (A2) should be $G_{C D}$.

${ }^{13}$ J. R. Macdonald, Phys. Rev. B 63, 052205 (2001).

${ }^{14}$ J. R. Macdonald, Solid State Ionics 150, 263 (2002)

${ }^{15}$ W. K. Lee, J. F. Liu, and A. S. Nowick, Phys. Rev. Lett. 67, 1559 (1991).

${ }^{16}$ J. R. Macdonald, J. Non-Cryst. Solids 307-310, 913 (2002).

${ }^{17}$ K. L. Ngai, J. Chem. Phys. 110, 10576 (1999).

${ }^{18}$ J. R. Macdonald, J. Chem. Phys. 115, 6192 (2001)

${ }^{19}$ J. R. Macdonald, Phys. Rev. B 66, 064305 (2002).

${ }^{20}$ J. R. Macdonald, J. Chem. Phys. 102, 6241 (1995)

${ }^{21}$ H. Scher and M. Lax, Phys. Rev. B 7, 4491 (1973).

${ }^{22}$ J. R. Macdonald, Inverse Probl. 16, 1561 (2000).

${ }^{23}$ C. T. Moynihan, Solid State Ionics 105, 175 (1998).

${ }^{24}$ K. L. Ngai, Philos. Mag. B 77, 187 (1998).

${ }^{25}$ J. R. Macdonald and L. D. Potter, Jr., Solid State Ionics 23, 61 (1987); J. R. Macdonald, J. Comput. Phys. 157, 280 (2000). The newest version of the comprehensive LEVM fitting program may be downloaded at no cost from http://www.physics.unc.edu/ macd/. It includes an extensive manual, executable programs, and full source code. More information is provided about LEVM at this www address.

${ }^{26}$ A. S. Nowick, A. V. Vaysleyb, and I. Kuskovsky, Phys. Rev. B 58, 8398 (1998).

${ }^{27}$ D. P. Almond, G. K. Duncan, and A. R. West, Solid State Ionics 8, 159 (1983).

${ }^{28}$ D. P. Almond, C. C. Hunter, and A. R. West, J. Mater. Sci. 19, 3236 (1984).

${ }^{29}$ E. F. Hairetdinov, N. F. Uvarov, H. K. Patel, and S. W. Martin, Phys. Rev. B 50, 13259 (1994).

${ }^{30}$ A. Ghosh and A. Pan, Phys. Rev. Lett. 84, 2188 (2000).

${ }^{31}$ J. R. Macdonald and G. B. Cook, J. Electroanal. Chem. 193, 57 (1985).

${ }^{32}$ S. R. Elliott, Solid State Ionics 27, 131 (1988). 
${ }^{33}$ C. León, M. L. Lucia, and J. Santamaria, Phys. Rev. B 55, 882 (1997).

${ }^{34}$ G. P. Johari and K. Pathmanathan, Phys. Chem. Glasses 29, 219 (1988).

${ }^{35}$ K. Pathmanathan and G. P. Johari, J. Chem. Phys. 95, 5990 (1991).

${ }^{36}$ K. Pathmanathan and G. P. Johari, J. Chem. Soc., Faraday Trans. 91, 329 (1994).

${ }^{37}$ J. R. Macdonald, Braz. J. Phys. 29, 332 (1999). Available for download at: http://www.sbf.if.usp.br/bjp/Vol29/Num2/v29_332.pdf.
${ }^{38}$ C. T. Moynihan, R. D. Bressel, and C. A. Angell, J. Chem. Phys. 55, 4414 (1971).

${ }^{39}$ M. Tatsumisago, C. A. Angell, and S. W. Martin, J. Chem. Phys. 97, 6968 (1992).

${ }^{40}$ D. L. Sidebottom, P. F. Green, and R. K. Brow, J. Non-Cryst. Solids 183, 151 (1995).

${ }^{41}$ K. L. Ngai, Phys. Rev. B 48, 13481 (1993).

${ }^{42}$ K. L. Ngai and C. León, Phys. Rev. B 60, 9396 (1999).

${ }^{43}$ K. L. Ngai and C. León, Solid State Ionics 125, 81 (1999). 